\title{
Real-World Treatment Patterns and Adherence to Oral Medication Among Patients with Bipolar Disorders: A Retrospective, Observational Study Using a Healthcare Claims Database
}

This article was published in the following Dove Press journal:

Neuropsychiatric Disease and Treatment

\author{
Takeshi Inoue (D) \\ Hiromi Sano ${ }^{2}$ \\ Yoshitsugu Kojima ${ }^{3}$ \\ Sakiko Yamada $\mathbb{D}^{3}$ \\ Osamu Shirakawa (iD ${ }^{4}$ \\ 'Department of Psychiatry, Tokyo \\ Medical University, Tokyo, Japan; ${ }^{2}$ Medical \\ Affairs, Otsuka Pharmaceutical Co., Ltd., \\ Osaka, Japan; ${ }^{3}$ Medical Affairs, Otsuka \\ Pharmaceutical Co., Ltd., Tokyo, Japan; \\ ${ }^{4}$ Department of Neuropsychiatry, Kindai \\ University Faculty of Medicine, Osaka, \\ Japan
}

Purpose: This study aimed to describe real-world treatment patterns and medication adherence among patients with bipolar disorder (BD) in Japan.

Patients and Methods: Adult patients with a BD diagnosis were identified between July 2013 and February 2018, using an employment-based health insurance claims database from the JMDC Inc. Treatment patterns of target drugs (mood stabilizers, antipsychotics) and adherence (measured by the proportion of days covered [PDC]) were assessed during the first- through third-year follow-up. Adherence was also assessed for patient subgroups.

Results: The analyzed population included 13,788 patients with BD. They were mostly prescribed sodium valproate, lithium, or aripiprazole (range: 21.1-27.4\%) across 3 years of follow-up, whereas lamotrigine was prescribed to $11.2-12.8 \%$ of patients. Benzodiazepines (70-87\%) and antidepressants (52-71\%) were commonly prescribed during all three followup periods. The mean PDC among all patients with BD was 0.51 during the first and increased to 0.61 during the third year. The mean PDC was 0.42 (first year) in patients aged $<30$ years and 0.49 in those aged $30-40$ years. The PDC was $0.44-0.61$ (depending on the drug class) in those who were prescribed a single-class target drug and $0.68-0.83$ in those prescribed two drug classes concomitantly.

Conclusion: This study documented generally low medication adherence among patients with $\mathrm{BD}$, and those at young age. These patients may require more attention.

Keywords: bipolar disorders, treatment patterns, medication adherence, oral antipsychotics, mood stabilizers, claims database

\section{Introduction}

Bipolar disorders (BD) are characterized by recurrent episodes of mania and depression. Although symptoms and prognosis vary among patients, approximately $70-80 \%$ of individuals diagnosed with BD experience a relapse in 4 to 5 years, ${ }^{1,2}$ while $10-15 \%$ experience more than 10 future episodes during their lifetime, ${ }^{3}$ and approximately up to $30 \%$ of patients have a rapid-cycling bipolar disorder. ${ }^{4}$ Relapses in patients with BD not only lower cognitive function and increase the risk of suicide, but are also associated with societal impacts. ${ }^{5}$ Although psychoeducation and psychotherapy are important, pharmacotherapy is considered a cornerstone of $\mathrm{BD}$ maintenance treatment to delay or prevent future episodes. However, non-adherence to prescribed medication is one of the greatest challenges
Correspondence: Sakiko Yamada Medical Affairs, Otsuka Pharmaceutica Co., Ltd., Shinagawa Grand Central Tower, 2-16-4 Konan, Minato-Ku, Tokyo, 108-8242, Japan

Tel $+8|367| 7 \mid 400$

Fax +81367161398

Email yamadasa@otsuka.jp
Neuropsychiatric Disease and Treatment 2021:17 821-833 
faced by clinicians in terms of therapeutic management of severe mental illnesses, including BD and schizophrenia.

Compared to other chronic conditions, adherence to medication therapy is particularly low in patients with BD and other psychiatric disorders, including schizophrenia. ${ }^{6}$ The proportion of days covered (PDC), ${ }^{7,8}$ a measure of adherence to medication therapy commonly used in studies analyzing administrative claims data, was $44-65 \%$ among patients with BD. ${ }^{9-11}$ Several factors have been reported as related to low adherence among patients with BD, including those patient related (eg, age, sex, distress associated with side effects, and believing medications are no longer needed) and therapy related (ie, the side effects of medication). ${ }^{6,12-16}$ Regardless of these factors, poor or no adherence to prescribed medications is an issue in patients with $\mathrm{BD}$ because it can negatively affect clinical outcomes (eg, relapse, symptoms, quality of life, hospitalizations, risk of suicide attempts, and healthcare costs). ${ }^{14,17}$ Hence, a better understanding of each of these risk factors may help design suitable interventions to improve adherence in individual patients. ${ }^{7}$

The guideline for the treatment of BD by the Japanese Society of Mood Disorder (JSMD) recommends lithium as the first-line maintenance treatment. ${ }^{18}$ The guideline also lists lamotrigine, olanzapine, quetiapine, aripiprazole, and paliperidone as the next recommended treatment options. To date, lamotrigine has been the only drug indicated for BD maintenance in Japan. However, to the best of our knowledge, reports on treatment patterns and adherence in patients with BD in clinical settings in Japan are sparse. Such information will be of benefit for clinicians in Japan.

To address this need, this study aimed to descriptively elucidate treatment patterns and medication adherence among patients with BD in clinical settings in Japan using a large-scale health insurance claims database. We also described adherence in patients with BD, stratified by baseline demographic, clinical characteristics, and target drugs (mood stabilizers, atypical antipsychotics, and typical antipsychotics). Atypical antipsychotics are also prescribed to patients with schizophrenia, and low adherence among these patients has been well reported. ${ }^{6}$ Treatment patterns and adherence among patients with schizophrenia were briefly examined in this study as a reference for patients with BD.

\section{Patients and Methods}

\section{Data Source and Study Design}

For this retrospective, observational cohort study, we used the health insurance claims data managed by the JMDC
Inc. (Tokyo, Japan) to identify patients with BD or schizophrenia. The patient data used for the current analysis were from July 1, 2012, to February 28, 2019. The JMDC database $^{19}$ includes de-identified data of medical (inpatient and outpatient) and pharmacy (dispensing) claims from approximately 7.4 million employees (aged $<75$ years) and their dependents, covering around $5 \%$ of the total population of Japan (as of August 2019). ${ }^{20}$ The database also includes data on health checkups. In addition to patient demographics, the database includes date-stamped claims information about inpatient and outpatient services (eg, diagnoses, procedures, prescriptions, medical services, costs, and medical institutions). Unless the employee withdraws from the participating health programs (ie, by quitting or changing jobs), the individual patient's medical and treatment history from multiple healthcare providers can be traced using the JMDC database.

As this retrospective observational study used a claims database compiled by the JMDC and did not collect new data, the Ethical Guidelines for Medical and Health Research Involving Human Subjects were not applicable. Because claims data provided by the JMDC were classified as anonymously processed information under the Act on the Protection of Personal Information 2003, informed consent from individual patients and reviews or approval from institutional review boards were not required.

\section{Study Sample}

Patients were included in the study if they were diagnosed with BD (according to the International Statistical Classification of Diseases and Related Health Problems, 10th revision [ICD-10] code: F30 or 31) recorded during the 365 days after the index date, between July 1, 2013, and February 1, 2018 (ie, the study period). The index date was defined as either (1) the earliest prescription date of the target drugs (mood stabilizers, atypical antipsychotics, and typical antipsychotics) listed in Supplementary Table 1 in the study period; or (2) when the target drugs were not identified, the earliest day of BD diagnosis in the study period. Patients were required to be at least 18 years of age at the index date and have not withdrawn from the participating insurance programs within 1 year after the index date. The prescription of target drugs was identified based on the treatment guidelines of the JSMD for $\mathrm{BD}^{18}$ (Supplementary Table 1).

Patients were excluded if they had recorded diagnosis/ es for schizophrenia (ICD 10 code: F20), dementia (F0003, F00-05, or G30-31), autism (F70 through F73, F79, or 
F84), or attention-deficit/hyperactivity disorder (F90) for 365 days before the index date. Patients with a diagnosis or prescription record of target drugs for BD 365 days before the index date (ie, between July 1, 2012, and the end of June 2013) during the study period were excluded (ie, only those patients who initiated pharmacological treatments at or after the index date were eligible).

The inclusion and exclusion criteria analogous to those for patients with $\mathrm{BD}$ were used to identify patients with schizophrenia (as a reference group), except that the diagnosis or prescription record of target drugs (atypical antipsychotics, typical antipsychotics, and others) were replaced for schizophrenia (Supplementary Table 2).

\section{Baseline Measures}

Baseline variables (only for patients with BD) assessed at the index date/month or 1 and 2 years after the index date/ month (ie, during the second- [366 days through 730 days] and third-year [731 days through 1095 days] follow-up) were the following: demographics (age, sex), depression (ICD-10 codes: F32 or F33), comorbidity score, member status, prescription of benzodiazepine drugs, antiParkinson drugs, antidepressants, or internal medicines, prescriptions of target drugs (yes or no), internal disorder, and hospital admission or outpatient visits (all departments or psychiatric). The comorbidity score was calculated based on the Charlson Comorbidity Index, ${ }^{21}$ but AIDS/ HIV was excluded due to data unavailability. Baseline variables assessed during the 365 days before the index date or the previous year included the comorbidity score, number and duration (in days) of hospital admission (all departments or psychiatry), and outpatient visits.

\section{Outcome Measures}

Treatment patterns were assessed during the first- (ie, from the index date through the first 365 days), second-, and third-year follow-up. During the study period, treatment patterns in terms of prescriptions of benzodiazepine drugs, anti-Parkinson drugs, antidepressants, or internal medicines, internal disorder, leave of absence (Supplementary Table 3), the number of outpatient visits, hospital admission (all or psychiatric), and the number of blood tests (Supplementary Table 3) were analyzed in patients with BD or schizophrenia. The treatment patterns of six commonly used drugs in Japan among the target drugs (ie, aripiprazole, olanzapine, quetiapine, sodium valproate, lamotrigine, and lithium) in terms of number of days prescribed, daily dose (in $\mathrm{mg}$ ), and the first dose of target drugs were analyzed only in patients with BD. The daily dose represented the estimated mean dose over the prescription period, and the first dose represented the initially dispensed dose.

Medication adherence was measured by PDC, which assesses the adherence to multiple medications prescribed in clinical practice where medications are switched or prescribed concomitantly with other drugs. Thus, the PDC is appropriate for patients with BD and other psychiatric disorders (eg, schizophrenia), to whom polypharmacy is often prescribed. The PDC ${ }^{22}$ was calculated as the total number of days' supply of any target drugs listed in Supplementary Table 1 divided by 365 days. Several target drugs prescribed on the same date were treated as one, but target drugs prescribed for one-off use were not counted when calculating the PDC. For the first year, the PDC was calculated for all patients; for the second- and third-year follow-up, it was calculated from patients who did not withdraw from the participating health programs and had a diagnosis record for $\mathrm{BD}$ or schizophrenia in each period.

\section{Statistical Analysis}

Descriptive statistics were used in all analyses in our study; baseline demographic and clinical characteristics (only for patients with BD) were summarized using the mean and standard deviation (SD) for the continuous variables, and the number and percentage for the categorical variables.

During the first-, second-, and third-year follow-up, the PDC was calculated separately for patients with BD (for those who were prescribed or not prescribed target drugs, and only for those who were prescribed these drugs) and schizophrenia as a reference. Similar calculations were made for subgroups of patients with BD, stratified by baseline demographic and clinical characteristics (those who were or were not prescribed target drugs), as well as for those who were prescribed target drugs. The PDC was also calculated for subgroups of patients with BD, stratified by the prescription of target drugs, with assessments made based on one drug class (ie, mood stabilizers, atypical antipsychotics, or antipsychotics only); two drug classes, but each with a single use (mood stabilizers and atypical antipsychotics); and two drug classes prescribed concomitantly, including the following three combinations: (1) mood stabilizers and atypical antipsychotics; (2) mood stabilizers only and mood stabilizers and atypical antipsychotics; and (3) atypical antipsychotics only and mood stabilizers and atypical antipsychotics; or other target drugs. All statistical 


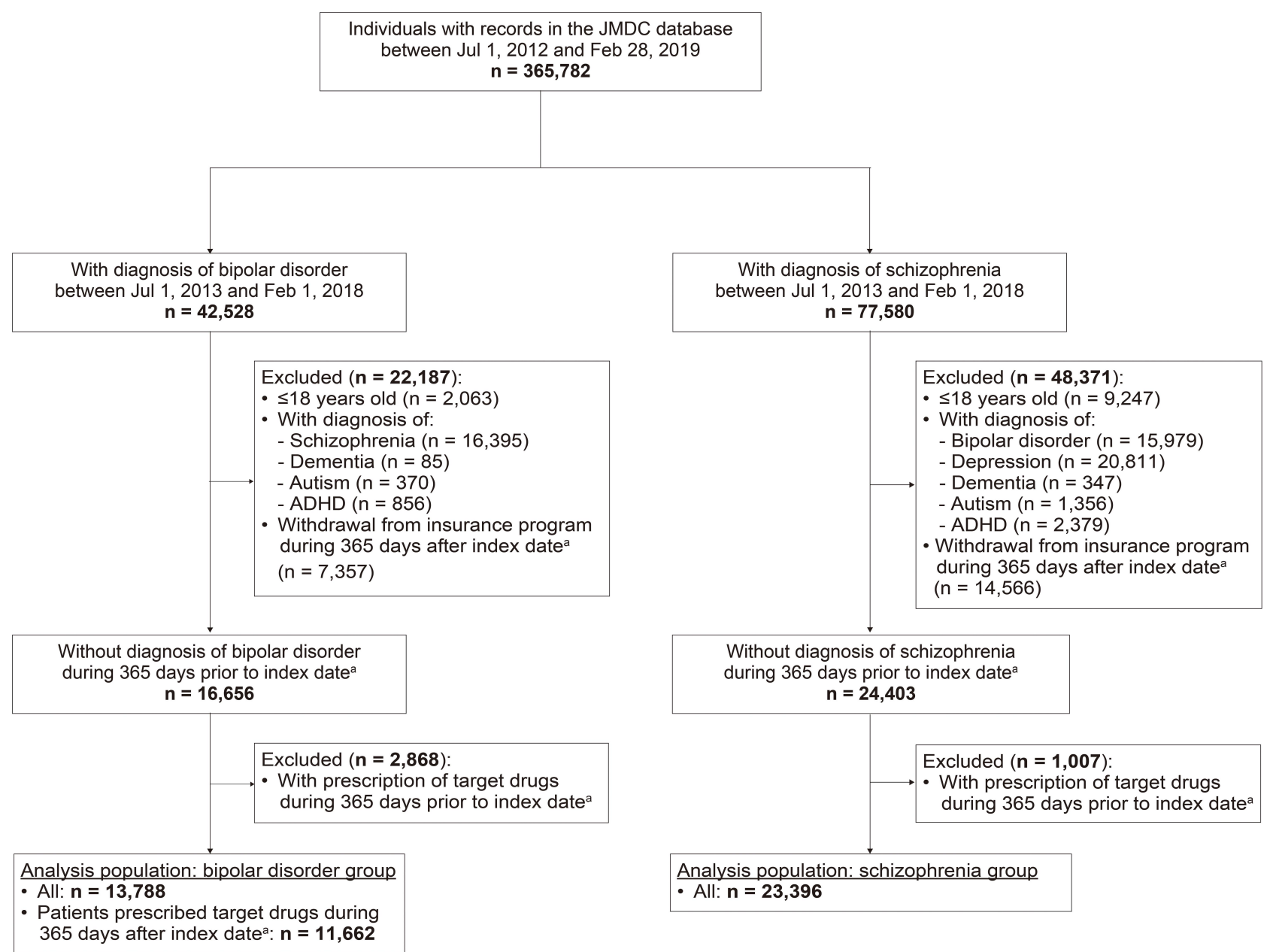

Figure I Patient disposition.

Notes: ${ }^{a}$ Index date for patients with BD was defined as either i) the earliest prescription date of target drugs (mood stabilizers, atypical antipsychotics, and typical antipsychotics) listed in Supplementary Table I in the study period (between July I, 2013 and February I, 20I8) or ii) the earliest day of BD diagnosis in the study period when the prescription date of the target drugs was not identified. The index date for patients with schizophrenia is analogous to that for BD, but the target drugs (atypical antipsychotics, typical antipsychotics, and others) are listed in Supplementary Table 2.

Abbreviations: ADHD, attention-deficit/hyperactivity disorder; BD, bipolar disorder

analyses were performed using the SAS version 9.4 (SAS Institute Inc., NC, USA).

\section{Results}

\section{Patient Selection, Baseline Demographic, and Clinical Characteristics}

Of the 365,782 individual patient records identified from the JMDC database, 42,528 patients had a BD diagnosis (Figure 1). A total of 13,788 patients were included in the final analysis population for BD, of which 11,662 (84.6\%) and $2126(15.4 \%)$, respectively, were and were not prescribed target drugs during the 365 days after the index date. In the schizophrenia group, 77,580 patients were diagnosed with schizophrenia, and 23,396 patients were included in the final analysis population.
The baseline demographic and clinical characteristics of all patients with BD during the first-, second-, and third-year follow-up are provided in Table 1. At the index date, the mean (SD) age of patients was 40.3 (11.6) years, and the proportion of female patients was $46.8 \%$. On the index date/month, 1 year, and 2 years after the index date/month, about half of the patients $(46.9 \%$, $46.2 \%$, and $45.2 \%$, respectively) were prescribed antidepressants. On the index date/month, 1 year, and 2 years after the index date/month, the target drugs were not prescribed to $21.5 \%, 35.0 \%$, and $35.2 \%$ of patients, respectively. Among the patients who were prescribed target drugs, the majority were prescribed mood stabilizers only $(42.6 \%, 34.1 \%$, and $32.8 \%$, respectively), followed by atypical antipsychotics only $(17.8 \%, 10.1 \%$, and $9.0 \%$, respectively). The mean (SD) number of 
Table I Baseline Demographic and Clinical Characteristics of Patients with BD During the First, Second, and Third Year of Follow-Up

\begin{tabular}{|c|c|c|c|}
\hline & First Year $(n=13,788)$ & Second Year $(n=5,494)$ & Third Year $(n=3,108)$ \\
\hline \multicolumn{4}{|c|}{ At the index date/month or I or 2 years after the index date/month } \\
\hline Age, years & $40.3(11.6)$ & $43.3(10.8)$ & $45.0(10.5)$ \\
\hline Female, n (\%) & $6453(46.8)$ & $2396(43.6)$ & $1298(4 \mid .8)$ \\
\hline Comorbidity (depression), n (\%) & $7216(52.3)$ & $2654(48.3)$ & $1498(48.2)$ \\
\hline \multicolumn{4}{|l|}{ Comorbidity score $^{a}, \mathrm{n}(\%)$} \\
\hline 0 & $11,05 \mid(80.1)$ & $434 I(79.0)$ & $2394(77.0)$ \\
\hline$>1$ & $2737(19.9)$ & $1153(21.0)$ & $7 \mid 4(23.0)$ \\
\hline \multicolumn{4}{|l|}{ Member status, $\mathrm{n}(\%)$} \\
\hline Member's dependents & $4696(34.1)$ & $1803(32.8)$ & $1022(32.9)$ \\
\hline Member & 9092 (65.9) & $3691(67.2)$ & $2086(67.1)$ \\
\hline \multicolumn{4}{|l|}{ Prescription, n (\%) } \\
\hline Benzodiazepine drugs, yes & $8739(63.4)$ & $3177(57.8)$ & $1808(58.2)$ \\
\hline Anti-Parkinson drugs, yes & $340(2.5)$ & $170(3.1)$ & $118(3.8)$ \\
\hline Antidepressants, yes & 6465 (46.9) & $2540(46.2)$ & $1404(45.2)$ \\
\hline Internal medicines, yes & 7955 (57.7) & $2804(51.0)$ & $1611(51.8)$ \\
\hline No prescription of target drugs ${ }^{\mathrm{b}}, \mathrm{n}(\%)$ & $2969(21.5)$ & $1922(35.0)$ & $1093(35.2)$ \\
\hline Hypnotics or anxiolytics only & $545(4.0)$ & $223(4.1)$ & $127(4.1)$ \\
\hline Antidepressants only & $381(2.8)$ & $207(3.8)$ & $106(3.4)$ \\
\hline Hypnotics or anxiolytics, plus antidepressants & $750(5.4)$ & $392(7.1)$ & $217(7.0)$ \\
\hline Others & $1293(9.4)$ & $1100(20.0)$ & $643(20.7)$ \\
\hline \multicolumn{4}{|l|}{ Prescription of target drugs ${ }^{\mathrm{b}}, \mathrm{n}(\%)$} \\
\hline Mood stabilizers only & $587 \mid(42.6)$ & $1873(34.1)$ & $1019(32.8)$ \\
\hline Atypical antipsychotics only & $2449(17.8)$ & $556(10.1)$ & $281(9.0)$ \\
\hline Conventional antipsychotics only & $346(2.5)$ & $109(2.0)$ & $70(2.3)$ \\
\hline Mood stabilizers and atypical antipsychotics & $1070(7.8)$ & $682(12.4)$ & $438(14.1)$ \\
\hline Mood stabilizers and conventional antipsychotics & $747(5.4)$ & $231(4.2)$ & $132(4.2)$ \\
\hline Others & $336(2.4)$ & $|2|(2.2)$ & $75(2.4)$ \\
\hline Internal disorder, yes, n (\%) & $3197(23.2)$ & $1386(25.2)$ & $916(29.5)$ \\
\hline \multicolumn{4}{|l|}{ Outpatients or inpatients, $\mathrm{n}(\%)$} \\
\hline Any departments (outpatients) & $13,510(98.0)$ & 5434 (98.9) & $3078(99.0)$ \\
\hline Any departments (inpatients) & $278(2.0)$ & $60(1.1)$ & $30(1.0)$ \\
\hline Psychiatry (outpatients) & I3,607 (98.7) & $5460(99.4)$ & $3087(99.3)$ \\
\hline Psychiatry (inpatients) & $181(1.3)$ & $34(0.6)$ & $21(0.7)$ \\
\hline \multicolumn{4}{|c|}{ During 365 days before the index date or the previous year } \\
\hline Comorbidity score $^{a}$ & $0.4(1.7)$ & $0.5(1.6)$ & $0.5(1.6)$ \\
\hline \multicolumn{4}{|l|}{ Hospital admission } \\
\hline Any departments, number & $0.0(0.2)$ & $0.1(0.3)$ & $0.1(0.3)$ \\
\hline Any departments, days & $0.3(4.4)$ & $2.1(12.4)$ & $2.2(12.5)$ \\
\hline Psychiatry, number & $0.0(0.1)$ & $0.0(0.2)$ & $0.0(0.2)$ \\
\hline Psychiatry, days & $0.0(0.0)$ & $0.0(0.0)$ & $0.0(0.0)$ \\
\hline Outpatient visits, number & $3.8(5.3)$ & $14.0(4.6)$ & I3.6 (4.8) \\
\hline
\end{tabular}

Notes: Data are expressed as mean (standard deviation) unless otherwise stated. ${ }^{2}$ Comorbidity score was calculated based on the Charlson Comorbidity Index (Quan et al, 2005) but excluded AIDS/HIV due to the unavailability of data. ${ }^{\text {b}}$ Target drugs (mood stabilizers, atypical antipsychotics, and typical antipsychotics) are listed in Supplementary Table I. Abbreviation: $\mathrm{BD}$, bipolar disorder. 
Table 2 Treatment Patterns and Clinical Characteristics in Patients with BD During the First, Second, and Third Year of Follow-Up

\begin{tabular}{|c|c|c|c|}
\hline & First Year $(n=13,788)$ & Second Year $(n=5,494)$ & Third Year $(n=3,108)$ \\
\hline \multicolumn{4}{|l|}{ Prescription, n (\%) } \\
\hline Benzodiazepine drugs, yes & $9671(70.1)$ & $460 \mathrm{I}(83.7)$ & $2702(86.9)$ \\
\hline Anti-Parkinson drugs, yes & $546(4.0)$ & $428(7.8)$ & $349(11.2)$ \\
\hline Antidepressants, yes & $7219(52.4)$ & $3680(67.0)$ & $2207(71.0)$ \\
\hline Internal medicines, yes & $8905(64.6)$ & $4323(78.7)$ & $2569(82.7)$ \\
\hline Internal disorder, yes, n (\%) & $4689(34.0)$ & $286 I(52.1)$ & $1914(61.6)$ \\
\hline Outpatient visits, number & $12.6(5.4)$ & I $3.3(4.8)$ & I $3.4(4.9)$ \\
\hline \multicolumn{4}{|l|}{ Hospital admission, n (\%) } \\
\hline Any departments, yes & $894(6.5)$ & $315(5.7)$ & $16 \mid(5.2)$ \\
\hline Psychiatry, yes & $423(3.1)$ & $143(2.6)$ & $85(2.7)$ \\
\hline Blood tests, number & $0.4(1.3)$ & $0.6(1.6)$ & $0.6(1.6)$ \\
\hline Leave of absence, $\mathrm{n}(\%)$ & $805(5.8)$ & $776(14.1)$ & $434(14.0)$ \\
\hline
\end{tabular}

Note: Data are expressed as mean (standard deviation) unless otherwise stated. Abbreviation: BD, bipolar disorder.

outpatient visits to clinics or hospitals during the 365 days before the index date was 3.8 (5.3), 14.0 (4.6), and 13.6 (4.8) times during the first, second, and third year, respectively.

\section{Treatment Patterns and Clinical}

\section{Characteristics}

A higher proportion of patients with $\mathrm{BD}$ were prescribed benzodiazepine drugs than those with schizophrenia during all three follow-ups, but the proportions in both groups increased from the first to the third year (first year: 70.1\% vs $60.9 \%$; second year: $83.7 \%$ vs $74.8 \%$; third year: $86.9 \%$ vs $79.0 \%$ ) (Table 2 and Supplementary Table 4). Antidepressants were prescribed to $52.4 \%$ of patients with $\mathrm{BD}$ during the first year, and the percentage increased during the second $(67.0 \%)$ and third $(71.0 \%)$ years, thus being prescribed more than to patients with schizophrenia (28.9\%, 37.1\%, and $40.1 \%$, respectively). Similarly, the proportion of patients with BD or schizophrenia who were prescribed anti-Parkinson drugs and internal medicines also increased from the first- to the third-year follow-up.

The mean (SD) number of outpatient visits to clinics or hospitals by patients with BD was $12.6(5.4), 13.3(4.8)$, and 13.4 (4.9) during the first-, second-, and third-year follow-up, respectively (Table 2), which was comparable with those by patients with schizophrenia (12.0 [5.3], 12.6 [5.1], and 12.7 [5.1] times, respectively) (Supplementary Table 4). The proportion of patients with BD admitted to psychiatric hospitals ranged from $2.6 \%$ to $3.1 \%$, depending on the year of follow-up, and it was lower than in those with schizophrenia $(6.2-8.2 \%)$.

\section{Treatment Patterns and Doses of Target Drugs in Patients with BD}

About one-quarter of patients with $\mathrm{BD}$ was prescribed aripiprazole (22.1-23.0\%), sodium valproate (21.7$24.0 \%$ ), or lithium (21.1-27.4\%) (Table 3). Lamotrigine was prescribed in $11.2-12.8 \%$ of patients with BD during the same period. During the first-year follow-up, the mean (SD) number of days with prescription of target drugs ranged from 118.6 (112.6) days for quetiapine to 224.0 (128.6) days for lithium; and the number of days was generally longer during the second- and third-year followup than during the first year, regardless of the target drug classes.

The mean (SD) daily dose of atypical antipsychotics was generally small, with aripiprazole, olanzapine, and quetiapine at 4.9 (6.6) $\mathrm{mg}, 8.1$ (13.4) $\mathrm{mg}$, and 93.5 (162.1) $\mathrm{mg}$, respectively, during the first-year follow-up. Lamotrigine was also prescribed in small doses, with a mean (SD) daily dose of 46.9 (29.2) $\mathrm{mg}$ during the firstyear follow-up. However, the mean (SD) daily doses of sodium valproate and lithium were 431.3 (221.3) $\mathrm{mg}$ and 490.0 (207.7) $\mathrm{mg}$, respectively, during the first-year follow-up. The daily dose of all target drugs changed little during all three follow-up periods. 
Table 3 Treatment Patterns and Doses of Six Commonly Prescribed Drugs in Patients with BD During the First, Second, and Third Year of Follow-Up

\begin{tabular}{|c|c|c|c|c|}
\hline \multirow[t]{2}{*}{ Drug } & \multirow[t]{2}{*}{ Parameter } & First Year $(n=13,788)$ & Second Year $(n=5,494)$ & Third Year $(n=3,108)$ \\
\hline & & Mean (SD) & Mean (SD) & Mean (SD) \\
\hline \multirow[t]{4}{*}{ Aripiprazole } & n (\%) & $3169(23.0)$ & $1212(22.1)$ & $700(22.5)$ \\
\hline & Number of days with prescription & $155.6(130.0)$ & $211.3(129.9)$ & $224.7(130.8)$ \\
\hline & Daily dose, mg & $4.9(6.6)$ & $5.1(6.0)$ & 5.9 (II.5) \\
\hline & Ist dose, $\mathrm{mg}$ & $5.1(8.7)$ & $5.3(7.6)$ & $6.1(11.2)$ \\
\hline \multirow[t]{4}{*}{ Olanzapine } & n (\%) & $1012(7.3)$ & $323(5.9)$ & $169(5.4)$ \\
\hline & Number of days with prescription & $126.6(121.5)$ & $186.0(132.8)$ & $202.1(138.1)$ \\
\hline & Daily dose, mg & $8.1(13.4)$ & $9.0(15.5)$ & $8.4(11.6)$ \\
\hline & Ist dose, $\mathrm{mg}$ & $8.3(13.8)$ & $9.6(16.0)$ & $8.6(11.8)$ \\
\hline \multirow[t]{4}{*}{ Quetiapine } & n (\%) & $659(4.8)$ & $332(6.0)$ & $208(6.7)$ \\
\hline & Number of days with prescription & $118.6(112.6)$ & $168.0(135.6)$ & I67.I (138.4) \\
\hline & Daily dose, mg & $93.5(162.1)$ & $99.3(144.4)$ & II $2.5(187.6)$ \\
\hline & Ist dose, $\mathrm{mg}$ & $91.3($ (I70.8) & I34.5 (329.0) & II $3.4(2 \mid 3.1)$ \\
\hline \multirow[t]{4}{*}{ Sodium valproate } & n (\%) & $3303(24.0)$ & $1255(22.8)$ & $675(21.7)$ \\
\hline & Number of days with prescription & $195.2(132.9)$ & $252.8(115.3)$ & $273.1(104.2)$ \\
\hline & Daily dose, mg & $431.3(221.3)$ & $475.8(249.2)$ & $497.5(254.9)$ \\
\hline & Ist dose, $\mathrm{mg}$ & $402.8(237.7)$ & $472.9(269.1)$ & $493.3(266.1)$ \\
\hline \multirow[t]{4}{*}{ Lamotrigine } & n (\%) & $1762(12.8)$ & $64 \mid(I I .7)$ & $348(11.2)$ \\
\hline & Number of days with prescription & I39.3 (I 23.6) & I88.5 (I27.8) & $203.4(130.8)$ \\
\hline & Daily dose, mg & $46.9(29.2)$ & $55.5(33.5)$ & $60.0(37.1)$ \\
\hline & Ist dose, $\mathrm{mg}$ & $35.8(30.3)$ & $50.8(38.4)$ & $57.0(52.4)$ \\
\hline \multirow[t]{4}{*}{ Lithium } & n (\%) & $2907(21.1)$ & $1385(25.2)$ & $853(27.4)$ \\
\hline & Number of days with prescription & $224.0(\mid 28.6)$ & $268.4(107.7)$ & $279.8(101.8)$ \\
\hline & Daily dose, mg & $490.0(207.7)$ & $534.0(221.9)$ & $537.7(220.9)$ \\
\hline & Ist dose, $\mathrm{mg}$ & 455.4 (268.6) & $535.4(276.2)$ & $541.2(238.8)$ \\
\hline
\end{tabular}

Note: The "daily dose" represents the mean daily dose over the prescription period, and "Ist dose" represents the first dose prescribed over the prescription period. Abbreviations: BD, bipolar disorder; SD, standard deviation.

\section{PDC Patterns for Overall Patients and Subgroups of Patients with BD}

The mean (SD) PDC for overall patients with BD was 0.51 $(0.40)$ in the first-year follow-up and increased subsequently $(0.60[0.40]$ and 0.61 [0.41] in the second and third year, respectively), and it was similar to those with schizophrenia (0.51 [0.42], 0.60 [0.42], and 0.61 [0.42], in the first-, second-, and third-year follow-up, respectively) (Table 4). Similar PDC patterns were observed for patients who were prescribed target drugs. The mean (SD) PDC in patients with $\mathrm{BD}$ was $0.61(0.37), 0.77(0.29)$, and 0.79 $(0.28)$ in the first-, second-, and third-year follow-up, and in patients with schizophrenia, it was $0.62(0.39), 0.79$ (0.29), and $0.80(0.28)$, respectively.

In the subgroups of patients with $\mathrm{BD}$, stratified by baseline demographic and clinical characteristics (those who were or were not prescribed target drugs), the mean (SD)
PDC was lower in patients aged $<30$ years and $30-40$ years than in patients aged $40-65$ years or $\geq 65$ years $(0.42$ [0.39] and $0.49[0.40]$ vs $0.56[0.40]$ and 0.55 [0.43], respectively) (Table 4). This pattern was also observed during the second and third year. The PDC was lower in women than in men during the first-year follow-up (0.49 [0.40] vs 0.54 [0.40]), and this pattern was also observed during the second- and third-year follow-up. The PDC was lower in patients with BD to whom antidepressants, benzodiazepine drugs, or antiParkinson drugs were not prescribed than in those to whom they were prescribed $(0.46[0.40]$ vs 0.57 [0.40]; 0.43 [0.40] vs 0.56 [0.40]; or 0.51 [0.40] vs 0.71 [0.37], respectively). This pattern was generally consistent during the second- and third-year follow-up. In general, these results were similar to those for subgroups of patients with BD stratified by baseline demographic and clinical characteristics who were prescribed target drugs only (Supplementary Table 5). 
Table 4 PDC for Patients with BD or Schizophrenia and for Subgroups of Patients with BD (Stratified by Baseline Demographic and Clinical Characteristics) During the First, Second, and Third Year of Follow-Up

\begin{tabular}{|c|c|c|c|c|c|c|}
\hline & \multicolumn{2}{|c|}{ First Year } & \multicolumn{2}{|c|}{ Second Year } & \multicolumn{2}{|c|}{ Third Year } \\
\hline & $\mathbf{n}$ & Mean (SD) & $\mathbf{n}$ & Mean (SD) & $\mathbf{n}$ & Mean (SD) \\
\hline \multicolumn{7}{|l|}{ Overall patients $^{\mathbf{a}}$} \\
\hline Bipolar disorder & 13,788 & $0.51(0.40)$ & 5494 & $0.60(0.40)$ & 3108 & $0.6 I(0.4 I)$ \\
\hline Schizophrenia & 23,396 & $0.51(0.42)$ & $10,27 \mid$ & $0.60(0.42)$ & 5771 & $0.61(0.42)$ \\
\hline \multicolumn{7}{|c|}{ Patients prescribed target drugs } \\
\hline Bipolar disorder & 11,662 & $0.61(0.37)$ & 4308 & $0.77(0.29)$ & 2405 & $0.79(0.28)$ \\
\hline Schizophrenia & 19,464 & $0.62(0.39)$ & 7859 & $0.79(0.29)$ & 4400 & $0.80(0.28)$ \\
\hline \multicolumn{7}{|c|}{ Subgroups of patients with BD $^{\mathbf{a}}$} \\
\hline \multicolumn{7}{|l|}{ Age, years } \\
\hline$<30$ & 2894 & $0.42(0.39)$ & 660 & $0.56(0.40)$ & 284 & $0.55(0.4 I)$ \\
\hline 30 to $<40$ & 3604 & $0.49(0.40)$ & 1314 & $0.55(0.4 I)$ & 666 & $0.56(0.42)$ \\
\hline 40 to $<65$ & 7095 & $0.56(0.40)$ & 3441 & $0.62(0.40)$ & 2104 & $0.64(0.4 I)$ \\
\hline$\geq 65$ & 195 & $0.55(0.43)$ & 79 & $0.66(0.43)$ & 54 & $0.62(0.4 I)$ \\
\hline \multicolumn{7}{|l|}{ Sex } \\
\hline Female & 6453 & $0.49(0.40)$ & 2396 & $0.57(0.4 \mathrm{I})$ & 1298 & $0.57(0.42)$ \\
\hline Male & 7335 & $0.54(0.40)$ & 3098 & $0.63(0.39)$ & 1810 & $0.64(0.40)$ \\
\hline \multicolumn{7}{|l|}{$\begin{array}{l}\text { Hospital admission (any } \\
\text { departments) }\end{array}$} \\
\hline No & 13,510 & $0.51(0.40)$ & 5434 & $0.60(0.40)$ & 3078 & $0.6 I(0.4 I)$ \\
\hline Yes & 278 & $0.51(0.42)$ & 60 & $0.63(0.42)$ & 30 & $0.43(0.46)$ \\
\hline \multicolumn{7}{|l|}{$\begin{array}{l}\text { Hospital admission } \\
\text { (psychiatry) }\end{array}$} \\
\hline No & 13,607 & $0.51(0.40)$ & 5460 & $0.60(0.40)$ & 3087 & $0.6 I(0.4 I)$ \\
\hline Yes & 181 & $0.62(0.39)$ & 34 & $0.70(0.39)$ & 21 & $0.44(0.46)$ \\
\hline \multicolumn{7}{|l|}{ Comorbidity score $^{\mathrm{b}}$} \\
\hline 0 & $|1,05|$ & $0.51(0.40)$ & 4341 & $0.60(0.40)$ & 2394 & $0.6 \mathrm{I}(0.4 \mathrm{I})$ \\
\hline$\geq 1$ & 2737 & $0.53(0.4 I)$ & 1153 & $0.60(0.4 I)$ & 714 & $0.61(0.42)$ \\
\hline \multicolumn{7}{|l|}{ Member status } \\
\hline Member's dependents & 4696 & $0.50(0.4 I)$ & 1803 & $0.59(0.4 \mathrm{I})$ & 1022 & $0.58(0.43)$ \\
\hline Member & 9092 & $0.52(0.40)$ & 3691 & $0.61(0.40)$ & 2086 & $0.63(0.40)$ \\
\hline \multicolumn{7}{|l|}{ Leave of absence } \\
\hline No & 13,327 & $0.51(0.40)$ & 5263 & $0.60(0.40)$ & 3040 & $0.6 I(0.4 I)$ \\
\hline Yes & 461 & $0.58(0.40)$ & 231 & $0.61(0.40)$ & 68 & $0.65(0.40)$ \\
\hline \multicolumn{7}{|l|}{ Depression } \\
\hline No & 6572 & $0.48(0.4 I)$ & 2840 & $0.57(0.4 \mathrm{I})$ & 1610 & $0.59(0.4 I)$ \\
\hline Yes & 7216 & $0.54(0.40)$ & 2654 & $0.63(0.40)$ & 1498 & $0.64(0.4 I)$ \\
\hline \multicolumn{7}{|l|}{ Prescriptions } \\
\hline \multicolumn{7}{|l|}{ Antidepressants } \\
\hline No & 7323 & $0.46(0.40)$ & 2954 & $0.56(0.4 I)$ & 1704 & $0.58(0.4 I)$ \\
\hline Yes & 6465 & $0.57(0.40)$ & 2540 & $0.64(0.40)$ & 1404 & $0.65(0.40)$ \\
\hline \multicolumn{7}{|l|}{ Benzodiazepine drugs } \\
\hline No & 5049 & $0.43(0.40)$ & 2317 & $0.51(0.40)$ & 1300 & $0.52(0.4 I)$ \\
\hline Yes & 8739 & $0.56(0.40)$ & 3177 & $0.66(0.39)$ & 1808 & $0.68(0.40)$ \\
\hline
\end{tabular}

(Continued) 
Table 4 (Continued).

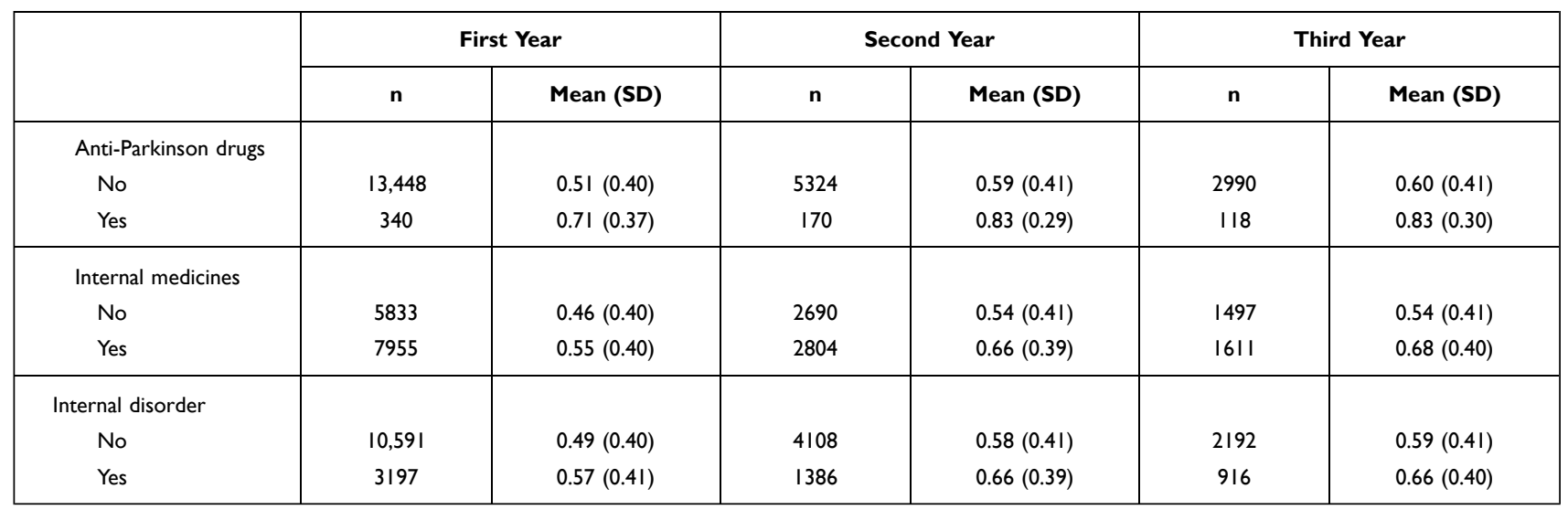

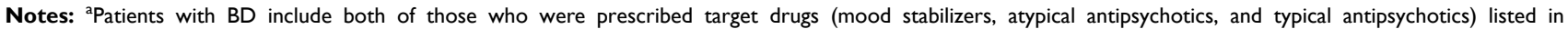
Supplementary Table I and those who were not prescribed these drugs; patients with schizophrenia include both of those who were prescribed drugs (atypical

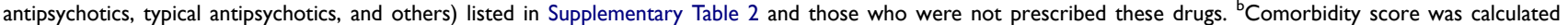
based on the Charlson Comorbidity Index ${ }^{21}$ but excluded AIDS/HIV due to the unavailability of data.

Abbreviations: PDC, proportion of days covered; BD, bipolar disorder; SD, standard deviation.

\section{PDC Patterns for Patients with BD (Stratified by Target Drugs)}

Among patients with $\mathrm{BD}$ who were or were not prescribed target drugs, the mean (SD) PDC in those who were prescribed mood stabilizers only, atypical antipsychotics, and typical antipsychotics was 0.61 (0.37), $0.46(0.38)$, and $0.44(0.37)$, respectively, in the first year (Table 5). The PDC for patients prescribed mood stabilizers and atypical antipsychotics separately was similarly low, with a mean (SD) PDC of 0.44 (0.28). In contrast, the PDC was generally higher in patients prescribed several drugs concomitantly, and the highest mean PDC (0.83 [0.24]) was found in those prescribed mood stabilizers only, and mood stabilizers and atypical antipsychotics concomitantly.

\section{Discussion}

We described the real-world treatment patterns and medication adherence among patients with $\mathrm{BD}$, by using a largescale, anonymized health insurance claims database in Japanese clinical settings. Among patients prescribed target drugs over three follow-ups, sodium valproate, lithium, or aripiprazole were the most commonly prescribed, and lamotrigine, the only medication indicated as BD maintenance therapy in Japan, was prescribed to approximately only $12 \%$ of patients. Patients with BD were commonly prescribed benzodiazepines (70-87\%) and antidepressants $(52-71 \%)$ in all three follow-ups. The mean PDC of 0.51 and 0.61 during the first and third year, respectively, for $\mathrm{BD}$ patients suggested that medication adherence was low in
Japan, and this low adherence was comparable to that of patients with schizophrenia, with a mean PDC of 0.51 and 0.61 . These values were lower than the adherence threshold of 0.80 , thus indicating non-adherence. ${ }^{10,11}$ The PDC was also low in younger patients aged $<30$ years across all three follow-ups.

Although the duration of prescription extended in lateryear follow-ups, the daily dose of atypical antipsychotics was relatively small during the first year and changed little over 3 years. For example, the estimated daily dose of aripiprazole in the first- and third-year follow-up was $4.9 \mathrm{mg}$ and $5.9 \mathrm{mg}$, respectively, both of which were smaller than the recommended dose of 12-24 mg for monotherapy or adjuvant therapy. ${ }^{23}$ The small daily dose of aripiprazole observed in our study may be because the manic or hypomanic episodes last only for $9 \%$ and $1 \%$ of the time in patients with BD I and II; $32 \%$ and $50 \%$ in those with depressive symptoms; $6 \%$ and $2 \%$ in those with cycling/mixed symptoms, respectively. ${ }^{24,25}$ Furthermore, a cross-sectional study in Japan reported that $39.7 \%$ of $\mathrm{BD}$ patients prescribed aripiprazole were in a depressive state $(24.9 \%)$ and remission $(14.8 \%)$, respectively, while only $14.6 \%$ were in a state of mania. ${ }^{26}$ Another possible reason for this small daily dose was that patients, especially insurance members, may have non-severer symptoms of mania. Members are employed and most of them are likely to be outpatients. Similar to aripiprazole, the daily dose of quetiapine found in our study was smaller than the recommended dose (50 mg initially and increased to $150 \mathrm{mg}$ after 2 days from the initial prescription ${ }^{27}$ ). This prescription pattern could be due to clinicians' concern over potential serious 
Table 5 PDC for Patients with BD Who Were and Were Not Prescribed Target Drugs Stratified by Prescription of Target Drugs ${ }^{\mathrm{a}}$ During the First, Second, and Third Year of Follow-Up

\begin{tabular}{|l|c|c|c|c|c|c|}
\hline \multirow{2}{*}{ Drugs } & \multicolumn{2}{|c|}{ First Year (n= I3,788) } & \multicolumn{2}{l|}{ Second Year (n= 5,494) } & \multicolumn{2}{c|}{ Third Year (n= 3, 108) } \\
\cline { 2 - 7 } & $\mathbf{n}(\%)$ & Mean (SD) & $\mathbf{n}(\%)$ & Mean (SD) & $\mathbf{n}(\%)$ & Mean (SD) \\
\hline Mood stabilizers only & $4766(34.6)$ & $0.61(0.37)$ & $1867(34.0)$ & $0.76(0.28)$ & $1046(33.7)$ & $0.80(0.25)$ \\
\hline Atypical antipsychotics only & $2159(15.7)$ & $0.46(0.38)$ & $604(11.0)$ & $0.67(0.33)$ & $314(10.1)$ & $0.67(0.35)$ \\
\hline Typical antipsychotics only & $348(2.5)$ & $0.44(0.37)$ & $121(2.2)$ & $0.61(0.34)$ & $84(2.7)$ & $0.56(0.39)$ \\
\hline $\begin{array}{l}\text { Mood stabilizers and atypical antipsychotics (each with } \\
\text { single use) }\end{array}$ & $191(1.4)$ & $0.44(0.28)$ & $35(0.6)$ & $0.47(0.29)$ & $19(0.6)$ & $0.51(0.27)$ \\
\hline $\begin{array}{l}\text { Mood stabilizers and atypical antipsychotics } \\
\text { (concomitant use) }\end{array}$ & $851(6.2)$ & $0.68(0.38)$ & $481(8.8)$ & $0.86(0.23)$ & $329(10.6)$ & $0.88(0.21)$ \\
\hline $\begin{array}{l}\text { Mood stabilizers only and mood stabilizers and atypical } \\
\text { antipsychotics (concomitant use) }\end{array}$ & $774(5.6)$ & $0.83(0.24)$ & $351(6.4)$ & $0.86(0.19)$ & $182(5.9)$ & $0.87(0.19)$ \\
\hline $\begin{array}{l}\text { Atypical antipsychotics only and mood stabilizers and } \\
\text { atypical antipsychotics (concomitant use) }\end{array}$ & $243(1.8)$ & $0.77(0.28)$ & $128(2.3)$ & $0.81(0.25)$ & $66(2.1)$ & $0.87(0.19)$ \\
\hline \begin{tabular}{l} 
Others \\
\hline
\end{tabular} & $4456(32.3)$ & $0.34(0.41)$ & $1907(34.7)$ & $0.29(0.42)$ & $1068(34.4)$ & $0.28(0.42)$ \\
\hline
\end{tabular}

Note: ${ }^{a}$ Target drugs (mood stabilizers, atypical antipsychotics, and typical antipsychotics) are listed in Supplementary Table I.

Abbreviations: PDC, proportion of days covered; BD, bipolar disorder; SD, standard deviation.

adverse events when used together with mood stabilizers (eg, valproic acid). ${ }^{28}$

Our findings suggest that the PDC among Japanese patients with $\mathrm{BD}$ who started oral pharmacotherapy was low (0.51-0.61), the result generally consistent with findings from previous studies using claims database (mean PDC of $0.65,,^{9} 0.45,{ }^{10}$ and $\left.0.44^{11}\right)$. The reason for a low PDC during the first year of follow-up is unknown, but these results indicate that patients initiating pharmacotherapy may require intervention to improve PDC. A large proportion of patients $(60 \%$ and $78 \%$, respectively) were lost to follow-up during the second and third year. Thus, it is speculated that some of these patients could be severely affected by their condition and might have lost their job and health insurance. Some of these patients may have also been lost to follow-up because their treatment journey extended beyond the study period, or they felt their condition improved and no longer required pharmacotherapy. Our results showed that the PDC improved in patients in the second- and third-year follow-ups $(0.60-0.79)$. Although this improvement needs to be carefully interpreted because the number of patients followed-up was small, it is possible that these patients might have severe conditions and required continuous and more intensive use of pharmacotherapy. In fact, the patients during the second and third year of follow-up visited hospitals slightly more often (the mean number of outpatient visits 13.3 and 13.4 times, respectively) than those during the first year (12.6 times). Their frequent visits to hospitals may also indicate that they were managing symptoms well (ie, experienced positive medications effects). ${ }^{29}$

Furthermore, our results suggested that BD patients who were prescribed two drug classes concomitantly presented a higher PDC (eg, 0.68-0.83 during the first year, $>0.80$ generally considered to indicate adherence). It has been speculated that patients who take multiple drugs would visit hospitals once every 2 weeks to a month by considering their condition, and they presumably understand the need for polypharmacy. In contrast, patients with $\mathrm{BD}$ who were prescribed one drug class at a time had a low PDC and may require intervention to improve the PDC. The improvement can, for example, shorten the time of remission, lower the risk of relapse and hospitalization, and improve the quality of life overall. ${ }^{14}$

Our result suggested that the PDC was low in younger patients ( 0.42 for $<30$ years), and this finding also supports previous studies according to which young age at $\mathrm{BD}$ onset ( $<30$ years at onset of affective episodes) is a risk factor for non-adherence to therapy. ${ }^{12}$ Younger patients may also have little knowledge of their condition, which may possibly lead to lower adherence. Thus, special attention may be required for optimizing maintenance therapy, 
especially in younger patients in Japan. In contrast to age, findings on the effects of sex on adherence were mixed, ${ }^{12,14,17,29}$ although it is also possible that a lower PDC in women may be related to concerns over the increased risk of side effects such as weight gain ${ }^{12,30}$ and teratogenicity associated with some medications (eg, valproate $\left.^{31}\right)$. The lower PDC of women in our study could probably be due to the higher representation of younger patients in the female subgroup than in the male subgroup: $53 \%$ of women were aged $<40$ years compared to $42 \%$ of men.

For patients with both BD and schizophrenia, ${ }^{6}$ and who had low adherence to orally administered antipsychotics, long-acting injectable (LAI) antipsychotics could be another treatment option. Previous studies have demonstrated the effectiveness of LAI antipsychotics on treatment continuation. For example, a real-world study using a large administrative claims database reported that BD patients treated with LAI antipsychotics (paliperidone, haloperidol, risperidone, aripiprazole, fluphenazine, or olanzapine) had a $5 \%$ higher PDC than those prescribed oral antipsychotic monotherapy. ${ }^{10}$ Furthermore, patients using oral antipsychotics were $19 \%$ more likely to discontinue treatment than those using LAI medication. ${ }^{10}$ In Japan, where LAI antipsychotics are still underused, a real-world study using the JMDC database demonstrated patients treated with LAI antipsychotics (ie, aripiprazole) were significantly less likely to discontinue treatment than those receiving aripiprazole orally (adjusted hazard ratio: $0.54 ; \mathrm{p}<0.0001){ }^{32}$ In the above studies, the mean age (range) of patients with BD who received LAI and oral antipsychotics was 36.1-38.4 years and 39.1-39.3 years, respectively, ${ }^{10,32}$ which was comparable with the age group of younger BD patients in our study (ie, $<30$ years or 30-40 years) who had poor medication adherence. Hence, the use of LAI antipsychotics could be an alternative treatment option, especially in younger patients.

\section{Limitations}

Our study has some limitations. First, the results may not be generalizable to the entire population with $\mathrm{BD}$ in Japanese clinical setting because (1) the claims data were obtained from society-managed, employment-based health insurance programs, which cover around $5 \%$ of the total population in Japan (as of August 2019); ${ }^{20}$ and (2) the employees aged $>65$ years were less represented in the database ( $3 \%$ of the total database population) compared with people aged $\geq 65$ years being represented in the
Japanese population $\left(28.1 \%\right.$ as of September 2019). ${ }^{33}$ Second, patients with non-severe symptoms associated with BD may be overrepresented. Because insurance members are employed, it is speculated that patients who were severely impacted by BD could lose their jobs and health insurance coverage and were not included in the database. However, such bias may not be present in family members. There were also limitations inherent to the claims database. Diagnostic codes and prescription records may be entered into the claims database for reimbursement purposes, and treatment patterns and PDC may overestimate actual medication intakes. The database did not contain information such as type of BD (I or II) and state of BD (eg, mania, depression, remission), attitudes of patients, disease severity, ${ }^{12}$ and socioeconomic status. Thus, prescription patterns and PDC were examined in the overall population. Further assessments may reinforce the understanding of PDC in patients with BD and identify patients in need of intervention. Finally, although PDC was examined in subgroups of patients with BD, causality between PDC and patients' background could not be established in this retrospective, observational study. Despite these limitations, owing to the large and valid sample collection of patient characteristics, the health insurance claims database could be a valuable source of information in a real-world setting.

\section{Conclusion}

In this large-scale, real-world retrospective, observational cohort study using the health insurance claims database, we documented that, in Japan, about one-quarter of patients with BD were prescribed sodium valproate, lithium, and aripiprazole, whereas lamotrigine, the only medication indicated for maintenance treatment for $\mathrm{BD}$, was prescribed to approximately only $12 \%$ of patients, with little changes in this treatment pattern over three yearly follow-ups. The data suggested that the PDC was possibly low in patients with BD during the first year following initiating pharmacotherapy, and especially low in younger patients. These patients may require special attention to improve their adherence and optimize maintenance therapy to improve clinical outcomes.

\section{Abbreviations}

$\mathrm{BD}$, bipolar disorders; PDC, proportion of days covered; JSMD, Japanese Society of Mood Disorder; ICD, International Statistical Classification of Diseases and 
Related Health Problems; SD, standard deviation; LAI, long-acting injectable.

\section{Data Sharing Statement}

The data that support the findings of this study are available for purchase from the JMDC Inc., and restrictions apply to the availability of these data due to contractual agreements between JMDC and health insurance association. For inquiries about access to the dataset used in this study, please contact JMDC (website, https://www.jmdc. co.jp/en/index; e-mail, mdbhelp@jmdc.co.jp).

\section{Ethics Approval and Informed Consent}

This retrospective observational study used de-identified claims database compiled by the JMDC and did not involve the collection of new data. The de-identified claims data were classified as anonymously processed information under the Act on the Protection of Personal Information 2003, and the Ethical Guidelines for Medical and Health Research Involving Human Subjects were not applicable to such data. Thus, informed consent from individual patients and reviews or approval from institutional review boards were not required.

\section{Consent for Publication}

Since the de-identified claims data provided by the JMDC Inc. were classified as anonymously processed information under the Act on the Protection of Personal Information 2003, informed consent from individual patients was not required.

\section{Acknowledgments}

We thank Dr. Tadashi Koga (Clinical Study Support, Inc.) for his assistance in the development of the statistical analysis plan. We thank Dr. Hidetsugu Tsubouchi (Otsuka Pharmaceutical Co., Ltd.) for editorial support. Medical writing assistance was provided by Clinical Study Support, Inc., and the support for statistical and medical assistance was funded by Otsuka Pharmaceutical Co., Ltd.

\section{Author Contributions}

All authors made substantial contributions to conception and design, acquisition of data, or analysis and interpretation of data; took part in drafting the article or revising it critically for important intellectual content; agreed to submit to the current journal; gave final approval of the version to be published; and agree to be accountable for all aspects of the work.

\section{Funding}

This work was sponsored by Otsuka Pharmaceutical Co., Ltd., Japan.

\section{Disclosure}

TI has received the following: personal fees from Mochida Pharmaceutical, Takeda Pharmaceutical, Eli Lilly, Janssen Pharmaceutical, MSD, Taisho Toyama Pharmaceutical, Yoshitomiyakuhin, and Daiichi Sankyo; grants from Shionogi, Astellas, Tsumura, and Eisai; and grants and personal fees from Otsuka Pharmaceutical Co. Ltd., Dainippon Sumitomo Pharma, Mitsubishi Tanabe Pharma, Kyowa Pharmaceutical Industry, Pfizer, Novartis Pharma, and Meiji Seika Pharma. TI is a member of the advisory boards of Pfizer, Novartis Pharma, and Mitsubishi Tanabe Pharma. OS has received speakers' honoraria from Eli Lilly Japan, Otsuka Pharmaceutical Co. Ltd., Otsuka Pharmaceutical Factory, Meiji Seika Pharma, Kyowa Pharmaceutical Industry, Pfizer Japan, Sumitomo Dainippon Pharma, Yoshitomiyakuhin, Alfresa Pharma, Eisai, and MSD. HS, YK, and SY are employees of Otsuka Pharmaceutical Co., Ltd., Japan. The authors report no other conflicts of interest in this work.

\section{References}

1. Gitlin MJ, Swendsen J, Heller TL, Hammen C. Relapse and impairment in bipolar disorder. Am J Psychiatry. 1995;152(11):1635-1640.

2. Simhandl C, König B, Amann BL. A prospective 4-year naturalistic follow-up of treatment and outcome of 300 bipolar I and II patients. J Clin Psychiatry. 2014;75(3):254-263. doi:10.4088/JCP.13m08601

3. Müller-Oerlinghausen B, Berghöfer A, Bauer M. Bipolar disorder. Lancet. 2002;359(9302):241-247. doi:10.1016/S0140-6736(02) 07450-0

4. Carvalho AF, Dimellis D, Gonda X, Vieta E, McLntyre RS, Fountoulakis KN. Rapid cycling in bipolar disorder: a systematic review. $J$ Clin Psychiatry. 2014;75(6):e578-586. doi:10.4088/ JCP.13r08905

5. Hong J, Reed C, Novick D, Haro JM, Windmeijer F, Knapp M. The cost of relapse for patients with a manic/mixed episode of bipolar disorder in the EMBLEM study. Pharmacoeconomics. 2010;28 (7):555-566. doi:10.2165/11535200-000000000-00000

6. García S, Martínez-Cengotitabengoa M, López-Zurbano S, et al. Adherence to antipsychotic medication in bipolar disorder and schizophrenic patients: a systematic review. J Clin Psychopharmacol. 2016;36(4):355-371. doi:10.1097/JCP.0000000000000523

7. Lam WY, Fresco P. Medication adherence measures: an overview. Biomed Res Int. 2015;2015:217047. doi:10.1155/2015/217047

8. Yamamoto T, Hyakuta TA. Japanese literature review of medication adherence assessments. Jpn Red Cross Hiroshima Coll Nurs. 2016;16:57-65. [in Japanese]. 
9. Bohlken J, Konrad M, Kostev K. Adherence to neuroleptic treatment in psychiatric practices: a retrospective study of 55 practices with more than 5000 bipolar and schizophrenic patients in Germany. Psychiatry Res. 2020;284:112758. doi:10.1016/j.psychres.2020.112758

10. Greene M, Yan T, Chang E, Hartry A, Touya M, Broder MS. Medication adherence and discontinuation of long-acting injectable versus oral antipsychotics in patients with schizophrenia or bipolar disorder. $J$ Med Econ. 2018;21(2):127-134. doi:10.1080/ 13696998.2017.1379412

11. Yan T, Greene M, Chang E, Hartry A, Touya M, Broder MS. Medication adherence and discontinuation of aripiprazole once-monthly $400 \mathrm{mg}$ (AOM 400) versus oral antipsychotics in patients with schizophrenia or bipolar i disorder: a real-world study using US claims data. Adv Ther. 2018;35(10):1612-1625 doi:10.1007/s12325-018-0785-y

12. Leclerc E, Mansur RB, Brietzke E. Determinants of adherence to treatment in bipolar disorder: a comprehensive review. J Affect Disord. 2013;149(1-3):247-252. doi:10.1016/j.jad.2013.01.036

13. Chakrabarti S. Treatment-adherence in bipolar disorder: a patient-centred approach. World J Psychiatry. 2016;6(4):399-409. doi:10.5498/wjp.v6.i4.399

14. Colom F, Vieta E, Tacchi MJ, Sánchez-Moreno J, Scott J. Identifying and improving non-adherence in bipolar disorders. Bipolar Disord. 2005;7(Suppl 5):24-31. doi:10.1111/j.1399-5618.2005.00248.x

15. Velligan DI, Weiden PJ, Sajatovic M, et al. The expert consensus guideline series: adherence problems in patients with serious and persistent mental illness. J Clin Psychiatry. 2009;70(Suppl 4):1-46. doi:10.4088/JCP.7090su1cj

16. Corréard N, Consoloni JL, Raust A, et al. Neuropsychological functioning, age, and medication adherence in bipolar disorder. PLoS One. 2017;12(9):e0184313. doi:10.1371/journal.pone.0184313

17. Bauer M, Glenn T, Alda M, et al. Trajectories of adherence to mood stabilizers in patients with bipolar disorder. Int $J$ Bipolar Disord. 2019;7(1):19. doi:10.1186/s40345-019-0154-z

18. Japanese Society of Mood Disorders. Treatment guidelines review committee for mood disorders: I. bipolar disorder 2020; 2020. Available from: https://www.secretariat.ne.jp/jsmd/iinkai/katsudou/data/guideline sokyoku2020.pdf. Accessed December 18, 2020. [in Japanese].

19. JMDC Inc. Tokyo. JMDC claims database; 2019. Available from: https://www.jmdc.co.jp/en/jmdc-claims-database. Accessed December 18, 2020. [in Japanese].

20. Japanese Society for Pharmacoepidemiology. Survey of Japanese databases in Japan available for clinical/pharmaco-epidemiology; 2019. Available from: http://www.jspe.jp/mt-static/FileUpload/files/ JSPE_DB_TF_E.pdf. Accessed December 18, 2020.

21. Quan H, Sundararajan V, Halfon P, et al. Coding algorithms for defining comorbidities in ICD-9-CM and ICD-10 administrative data. Med Care. 2005;43(11):1130-1139. doi:10.1097/01. mlr.0000182534.19832.83
22. Nau DP. Proportion of days covered (PDC) as a preferred method of measuring medication adherence; 2012. Available from: http://ep. yimg.com/ty/cdn/epill/pdcmpr.pdf. Accessed December 18, 2020.

23. ABILIFY ${ }^{\circledR}$ (aripiprazole) [package insert]. Tokyo; Otsuka; 2020. Available from: https://www.pmda.go.jp/PmdaSearch/iyakuDetail/ ResultDataSetPDF/180078 1179045F4022 1 19. Accessed September 20, 2000. [in Japanese].

24. Judd LL, Akiskal HS, Schettler PJ, et al. A prospective investigation of the natural history of the long-term weekly symptomatic status of bipolar II disorder. Arch Gen Psychiatry. 2003;60(3):261-269. doi:10.1001/archpsyc.60.3.261

25. Judd LL, Akiskal HS, Schettler PJ, et al. The long-term natural history of the weekly symptomatic status of bipolar I disorder. Arch Gen Psychiatry. 2002;59(6):530-537. doi:10.1001/archpsyc.59.6.530

26. Tsuboi T, Suzuki T, Azekawa T, et al. Factors associated with non-remission in bipolar disorder: the multicenter treatment survey for bipolar disorder in psychiatric outpatient clinics (MUSUBI). Neuropsychiatr Dis Treat. 2020;16:881-890. doi:10.2147/NDT. S246136

27. Bipresso ${ }^{\circledR}$ (quetiapine) [prescribing information]; 2020. Available from: https://pins.japic.or.jp/pdf/newPINS/00066992.pdf. Accessed September 29, 2020. [in Japanese].

28. Vella T, Mifsud J. Interactions between valproic acid and quetiapine/ olanzapine in the treatment of bipolar disorder and the role of therapeutic drug monitoring. J Pharm Pharmacol. 2014;66(6):747-759. doi:10.1111/jphp.12209

29. Vega P, Barbeito S, de Azúa SR. Bipolar disorder differences between genders: special considerations for women. Womens Health (Lond). 2011;7(6):663-674. doi:10.2217/WHE.11.71

30. McIntyre RS. Understanding needs, interactions, treatment, and expectations among individuals affected by bipolar disorder or schizophrenia: the UNITE global survey. J Clin Psychiatry. 2009;70 (Suppl 3):5-11. doi:10.4088/JCP.7075su1c.02

31. Viguera AC, Cohen LS, Baldessarini RJ, Nonacs R. Managing bipolar disorder during pregnancy: weighing the risks and benefits. Can $J \quad$ Psychiatry. 2002;47(5):426-436. doi:10.1177/0706743702 04700503

32. Iwata N, Inagaki A, Sano H, Niidome K, Kojima Y, Yamada S. Treatment persistence between long-acting injectable versus orally administered aripiprazole among patients with schizophrenia in a real-world clinical setting in Japan. Adv Ther. 2020;37 (7):3324-3336. doi:10.1007/s12325-020-01396-w

33. Statistics of Japan. Estimated population in February 2019; 2019 Available from: https://www.stat.go.jp/data/jinsui/pdf/201902.pdf. Accessed July 13, 2020. [in Japanese].
Neuropsychiatric Disease and Treatment

\section{Publish your work in this journal}

Neuropsychiatric Disease and Treatment is an international, peerreviewed journal of clinical therapeutics and pharmacology focusing on concise rapid reporting of clinical or pre-clinical studies on a range of neuropsychiatric and neurological disorders. This journal is indexed on PubMed Central, the 'PsycINFO' database and CAS, an is the official journal of The International Neuropsychiatric Association (INA). The manuscript management system is completely online and includes a very quick and fair peer-review system, which is all easy to use. Visit http://www.dovepress.com/testimonials.php to read real quotes from published authors. 